\title{
Ground- and space-based study of two globular cluster cataclysmic variables: M22 CV1 and M5 V101
}

\author{
A. P. Hourihane, ${ }^{1 \star}$ P. J. Callanan, ${ }^{1}$ A. M. Cool $^{2}$ and M. T. Reynolds ${ }^{3}$ \\ ${ }^{1}$ Department of Physics, University College Cork, Cork, Ireland \\ ${ }^{2}$ Department of Physics and Astronomy, San Francisco State University, 1600 Holloway Avenue, San Francisco, CA 94132, USA \\ ${ }^{3}$ Department of Astronomy, University of Michigan, 500 Church Street, Ann Arbor, MI 48109, USA
}

Accepted 2011 January 14. Received 2011 January 14; in original form 2010 November 29

\begin{abstract}
As a class of compact binaries with large binding energy, cataclysmic variables (CVs) formed through close encounters play an important role in the dynamical evolution of globular clusters. As part of a systematic search for CVs undergoing dwarf nova (DN) eruptions in globular clusters, our 2004 monitoring programme of M22 detected an outburst of the DN candidate CV1 during May. We implement the Isis image subtraction routine to obtain a light curve for an outburst of CV1. We present the outburst light curve as well as Hubble Space Telescope/WideField Planetary Camera 2 (WFPC2) photometry in the $V, U$ and near-ultraviolet $(n U V)$ bands and a Chandra/Advanced CCD Imaging Spectrometer (ACIS) spectrum of the object. Our results confirm the DN nature of the outburst and the CV status of the object. We also present the results of a ground-based study of another globular cluster CV, M5 V101 - including quiescent medium-resolution William Herschel Telescope (WHT)/Intermediate dispersion Spectrograph and Imaging System (ISIS) spectroscopy in the $B$ and $R$ bands, displaying prominent Balmer and $\mathrm{He}$ I emission, and $R$-band photometry.
\end{abstract}

Key words: techniques: photometric - stars: dwarf novae $-\mathrm{X}$-rays: binaries.

\section{INTRODUCTION}

Compact binary systems in globular clusters (GCs) are an important target for observations. Many of these systems, unlike primordial field binaries, are thought to be formed dynamically - for example, via three-body encounters and exchange interactions (e.g. Hut \& Verbunt 1983; Ivanova et al. 2006) - often leading to very close binaries, possibly with different properties relative to their counterparts in the field. Cataclysmic variables (CVs) are compact binaries comprising a white dwarf primary accreting from a low-mass Roche lobe filling secondary companion, usually through an accretion disc. An increase in mass transfer through the disc can lead to a brightening of the system of several magnitudes, known as a dwarf nova (DN) eruption.

So far, relatively few DN outbursts of GC CVs have been observed (e.g. Shara et al. 1996; Ó Tuairisg et al. 2003; Pietrukowicz et al. 2008) compared to the predicted populations of such systems. Ivanova et al. (2006) predict from numerical simulations that the number of CVs per unit mass should be 2-3 times higher in GC cores than in the field. Pietrukowicz et al. (2008) estimate the number of erupting $\mathrm{CVs}$ per unit mass in the solar neighbourhood to be $1.9-4.8 \times 10^{-5}$ per $\mathrm{M}_{\odot}$. However, they find that the number of CVs per unit mass in GCs which have been observed to undergo

${ }^{\star}$ E-mail: a.hourihane@ucc.ie
DN eruptions is significantly ( $\sim 10$ times) smaller than in the local field. It is likely that selection effects play a role in this apparent discrepancy. For example, Gänsicke (2005) notes that out of 531 field CVs with known orbital periods, those systems discovered through their variability are comprised of 86 per cent classical and dwarf novae $(\mathrm{DNe})$, whereas more than one half of the $121 \mathrm{X}$-ray selected systems in the sample are magnetic systems, which erupt relatively infrequently, if at all. On the other hand, it is plausible that despite the role of selection effects, the observational evidence for a lack of outbursts in GC CVs nevertheless reflects an underlying difference in the nature of the cluster CVs compared to field CVs.

As well as the bright GC X-ray sources $\left(L_{\mathrm{X}} \gtrsim 10^{36} \mathrm{erg} \mathrm{s}^{-1}\right)$ which have been identified with low-mass X-ray binaries (LMXBs; Grindlay et al. 1984), a population of fainter $\left(L_{\mathrm{X}} \lesssim 10^{33} \mathrm{erg} \mathrm{s}^{-1}\right)$ $\mathrm{X}$-ray sources in a number of GCs has been more recently identified as being composed of a significant proportion of CVs. Using the Hubble Space Telescope (HST), Cool et al. (1995) optically identified the first sample of CVs in a GC, coinciding with a population of ROSAT X-ray sources in NGC6397 (Cool et al. 1993) the current number of $\mathrm{CV}$ identifications in the cluster is $\sim 15$ (Cohn et al. 2010). Meanwhile, the largest sample of CVs (22) in a GC has been identified in 47 Tuc (for a list of the observations, see Heinke et al. 2005). This number compares favourably (within a factor of 2) to the theoretical prediction of Ivanova et al. (2006) of 35-40 detectable CVs (and up to 200 total CVs) in a 47 Tuc-like $\mathrm{GC}$ (where observational limits for field CVs are imposed to define 
detectability). The confirmed CV populations in these and other GCs allow us to compare the properties of these predominantly dynamically formed GC CVs to those of primordial field CVs and also to theoretical models.

It appears there is some evidence for a $\mathrm{CV}$ population in GCs with different characteristics to the field population. Many GC CVs have higher X-ray luminosities than field CVs (Verbunt et al. 1997), for instance. Many also exhibit X-ray to optical flux ratios higher than those typically seen in field CVs (this was found in the case of the 47 Tuc CVs by Edmonds et al. 2003b), indicative of DNe but in contradiction to the aforementioned dearth of observed DN eruptions. Furthermore, the presence of He II emission lines in the HST spectra of some GC CVs suggests a possible magnetic nature (as proposed for CVs in NGC 6397 by Grindlay et al. 1995).

These GC CVs are difficult to observe from the ground in their quiescent state due to their intrinsic faintness and their crowded location in the densely populated cluster cores. GC CV candidates have thus far typically been identified on the basis of their X-ray or far-UV emission (with optical follow-up observations needed for confirmation) and, in fewer instances, the presence of the more definitive DN outbursts. While the resolving power of $H S T$ is needed for the detailed optical photometric or spectral analysis necessary for conclusive classification of these crowded sources, the advent of image-subtraction software such as ISIs (Alard \& Lupton 1998; Alard 2000) allows us to search for eruptions of these systems from the ground.

CV1 is located in the crowded central region of the nearby (3.2 kpc; Harris 1996) GC, M22. Previously, Sahu et al. (2001) interpreted a $\sim 3$ mag brightening of the source in 1999 May as gravitational microlensing of a background bulge star by a low-mass foreground cluster object. Subsequent analysis of $H S T$ archival data by Anderson, Cool \& King (2003), including proper-motion studies confirming the object as a cluster member, prompted them to reclassify the object as a cluster $\mathrm{CV}$ which had undergone a DN eruption during the observations by Sahu et al. (2001). They also found that, in optical colours, the star is unusually red for a $\mathrm{CV}$, with $(B-R)$ and $(V-I)$ colours about 0.2 and 0.1 mag redwards of the main sequence, respectively. Bond et al. (2005) reported two outbursts of the object occurring during 2002-2003 from ground-based $I$-band observations during that epoch. The outburst amplitudes were $\Delta I=$ 2-3 mag and the full width at half-maximum of the duration (measurable for one outburst only) was approximately $14 \mathrm{~d}$. In another ground-based study, Pietrukowicz et al. (2005) reported a further two outbursts of CV1 - one occurring in 2000 August/September, with a duration of $\sim 20 \mathrm{~d}$ and the other event, for which they had incomplete data coverage, in 2001 June. More recently, Webb et al. (2004) have suggested that CV1 may be not be a CV, but could instead be a quiescent LMXB that exhibits outbursts. We studied M22 CV1 with the aim of placing a new constraint on the outburst frequency as well as further exploring the broad-band optical colours and investigating the X-ray properties to see if a quiescent LMXB ( $q L M X B$ ) identification could be ruled out for the source. We report on a long-term ground-based optical monitoring programme of M22 during which an outburst of CV1 was detected, and we also present archival HST photometry and analysis of the X-ray properties of the object.

Although the DN V101 lies at the greater distance of $7.5 \mathrm{kpc}$ from us in the GC M5 (Harris 1996), its atypical location 10 core radii from the centre of the cluster and lack of close neighbours allow it to be studied spectroscopically from the ground. A review of the available literature on V101 was given by Neill et al. (2002). From ground-based $I$-band observations, they determined the orbital period of the system to be $P_{\text {orb }}=5.796 \pm 0.036 \mathrm{~h}-$ the first orbital period determination for a DN in a GC. The only literature on the source since that time is the report by Pietrukowicz et al. (2008) of the detection of two new outbursts in 2003 and 2004 as part of their Cluster AgeS Experiment (CASE), as well as two previous outbursts in 1997, which had already been reported by Kaluzny et al. (1999) in their ground-based study of the cluster. We present an optical multiwavelength study of the M5 DN, V101, in an attempt to confirm its orbital period and identify the spectral type of the secondary.

\section{OBSERVATIONS AND DATA ANALYSIS}

\subsection{M22: optical photometry and X-ray spectroscopy}

\subsubsection{Ground-based optical photometry}

The M22 ground-based data set consists of $V$ - and $B$-band images covering the period of 2004 March-November, taken with the Small and Moderate Aperture Research Telescope System (SMARTS) 1.3-m telescope at the Cerro Tololo Inter-American Observatory (CTIO), Chile. The $1024 \times 1024$ pixel ANDICAM detector used for the observations has a pixel scale of $0.369 \operatorname{arcsec}_{\text {pixel }}^{-1}$ and a $6 \times$ $6 \mathrm{arcmin}^{2}$ field of view. The images are centred on the core of the GC with a sampling of approximately two 300-s $V$-band exposures every second night for most of the observing programme, increasing during May to two exposures per night in both the $V$ and $B$ bands as soon as the outbursting object was discovered.

Due to severe crowding effects at the cluster core and the faintness of the CV (see Figs 1 and 2), standard photometric reduction techniques such as aperture and profile-fitting photometry could not be performed on the source. For example, even small aperture photometry would be contaminated by light from neighbouring stars. Also posing a problem was the uncertain level of the sky background, which can vary quite strongly in such crowded regions due to, for

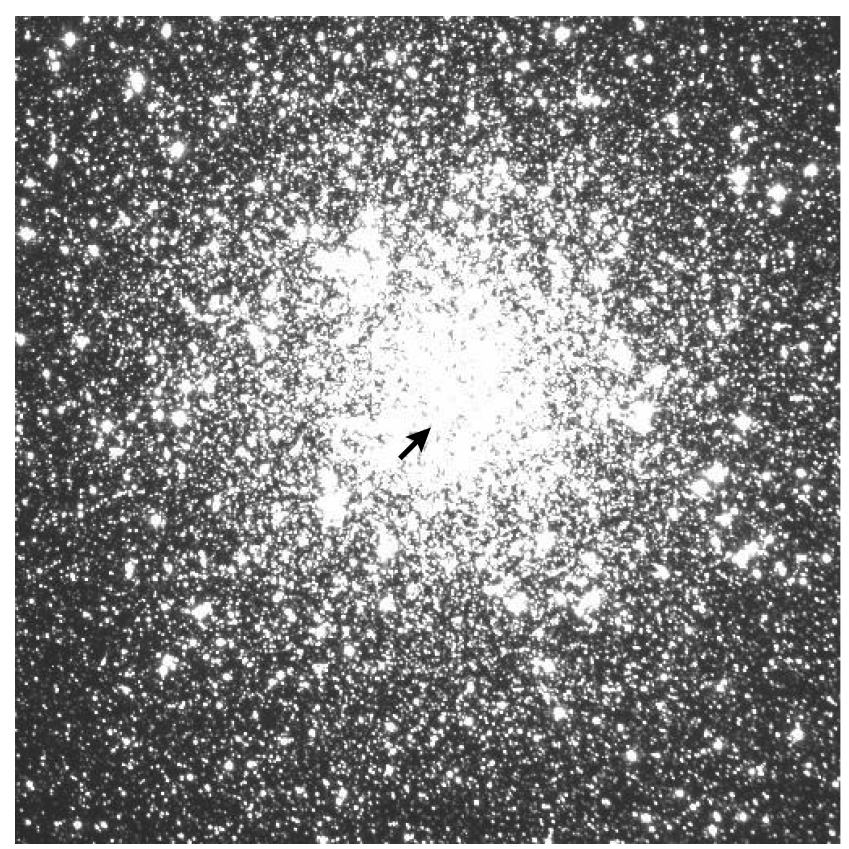

Figure 1. One of our SMARTS $V$-band images of M22 showing the location of CV1 near the centre of the cluster. The image clearly illustrates the crowded nature of this field. 

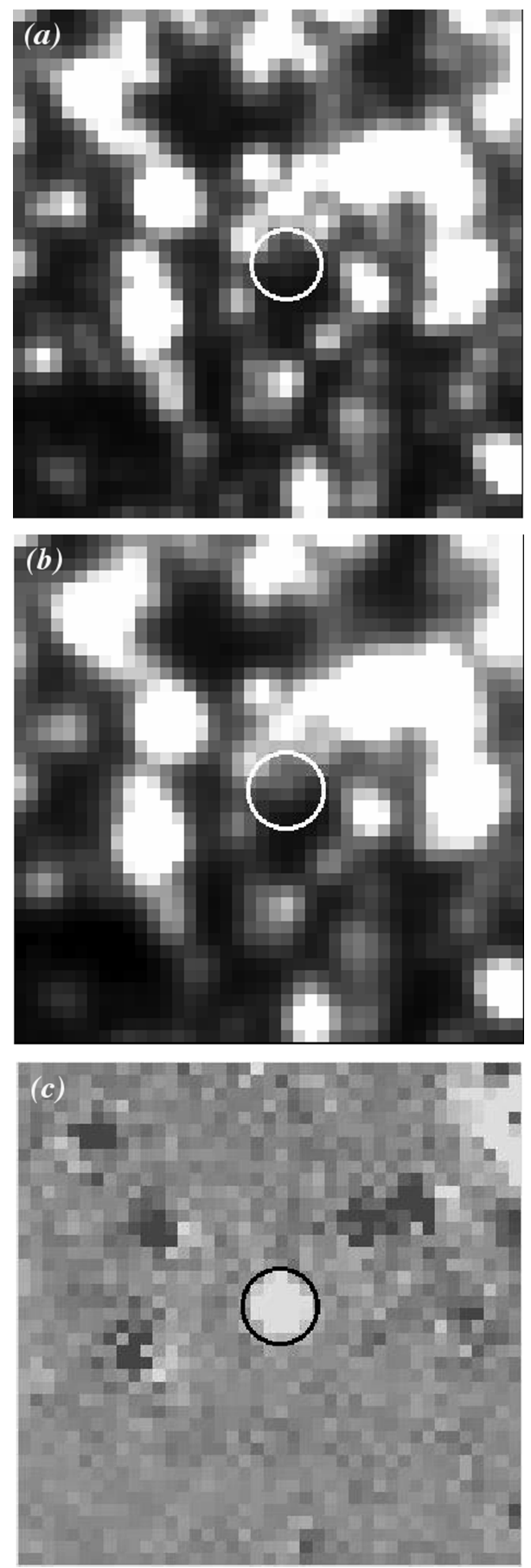

Figure 2. SMARTS $V$-band image sections centred on M22 CV1. (a) An image from the night of 2004 April 17 shows the location of the quiescent CV; (b) shows the CV in outburst on 2004 May 13. In (c), CV1 is clearly visible in outburst during 2004 May in a subtracted image processed with the ISIs software. Residuals from saturated neighbouring stars also appear in this image.

one, faint unresolved background stars. Moreover, the crowded nature of the entire image meant it was impossible to construct a model point spread function (PSF) of sufficient quality for profile-fitting photometry. The photometry was instead performed using the ISIS image subtraction software (Alard \& Lupton 1998; Alard 2000). Using the programs in this package, the images were first aligned with each other by remapping on to a common grid. A reference image
Table 1. List of archival space mission data used in the analysis.

\begin{tabular}{|c|c|c|c|c|}
\hline Satellite & Instrument & ID & Date & Exp. time \\
\hline$H S T$ & WFPC $2 / F 555 W$ & U9BM0704M & 2006 April 01 & 30 \\
\hline$H S T$ & WFPC $2 / F 336 W$ & U9BM0703M & 2006 April 01 & 500 \\
\hline$H S T$ & WFPC $2 / F 255 W$ & U9BM0701M-702M & 2006 March 31 & 800 \\
\hline Chandra & ACIS-S & 5437 & 2005 May 24 & 16020 \\
\hline
\end{tabular}

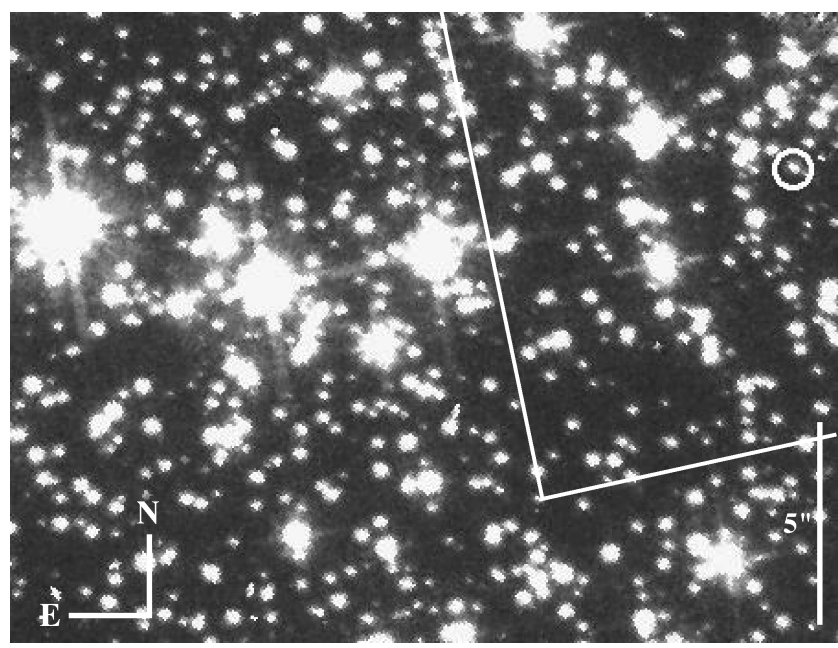

Figure 3. Finding chart for M $22 \mathrm{CV} 1$, taken from archival HST image $\mathrm{u} 9 \mathrm{bm} 0704 \mathrm{~m}(F 555 \mathrm{~W})$, showing the region of overlap with the SMARTS images in Fig. 2. The quiescent CV appears at the edge of chip WF4 of the WFPC2.

for subtraction was created by combining the 10 per cent of the images with the best seeing. This reference image was then convolved with a spatially varying kernel to degrade its seeing to match the seeing of each individual image in turn. Each individual image was then subtracted from the appropriately convolved reference image, with the result of creating a subtracted image containing the residuals (the outbursting CV is obvious in these subtracted images see Fig. 2c). All the subtracted images were median-combined to produce an image, var.fits, showing - in theory - only the variable stars, which appear as stellar-like profiles. In practice, saturated stars and cosmic rays lead to many spurious detections. The light curve of the $\mathrm{CV}$ was extracted from the subtracted images in the $V$ and $B$ bands using the PHOT routine packaged with ISIS and plotted in units of differential flux relative to the reference image in which the $\mathrm{CV}$ was in quiescence.

\subsubsection{HST data}

We retrieved archival multicolour HST images of M22 from the Multimission Archive at STScI. ${ }^{1}$ A list of the observations is given in Table 1. CV1 appears on chip WF4 of the Wide-Field Planetary Camera 2 (WFPC2), as seen in Fig. 3: its coordinates are $\alpha=18^{\mathrm{h}} 36^{\mathrm{m}} 24^{\mathrm{s}} .66, \delta=-23^{\circ} 54^{\prime} 35^{\prime \prime} 5$ (J2000; Sahu et al. 2001). In order to correct the counts in each pixel of the pipeline-calibrated

\footnotetext{
${ }^{1}$ Based on observations made with the NASA/ESA $H S T$, obtained from the data archive at the Space Telescope Science Institute (STScI). STScI is operated by the Association of Universities for Research in Astronomy, Inc. under NASA contract NAS 5-26555.
} 
images for the effects of geometric distortion (which appears at the 1-2 per cent level near the edges of the images and up to a maximum of 4-5 per cent in the corners, for fixed-aperture photometry), we multiplied each image by the correction image obtained from the archive. Cosmic rays were removed in IRAF ${ }^{2}$ with the STSDAS CRREJ tool for the co-aligned images taken with the $F 255 \mathrm{~W}$ filter $\left(n U V_{255^{-}}\right.$ band) and with the L.A.COSMIC Laplacian edge-detection routine (van Dokkum 2001) for the individual images in the $F 336 \mathrm{~W}$ and $F 555 \mathrm{~W}$ filters ( $U_{336^{-}}$and $V_{555}$-band, respectively). We performed smallaperture photometry in the $U_{336}$ and $V_{555}$ bands with the DAOPHOT (Stetson 1987) package in IRAF on all objects detected - including CV1 - in the $V_{555}$-band image on the PC, WF2 and WF4 chips. We corrected the results to the aperture for which the zeropoint was defined. The magnitudes were calibrated on the STMAG flux-based system using the zero-point calculated from the SYNPHOT PHOTFLAM header keyword. All the magnitudes were corrected for charge transfer efficiency losses using the correction formulae of Andy Dolphin, ${ }^{3}$ and the UV measurements were also corrected for the reduction in throughput due to the build-up of contaminants on the UV filters in between the regular decontaminations.

For CV1, we also calculated the STMAG $n U V_{255}$ magnitude and the $\left(n U V_{255}-U_{336}\right)$ colour. The results for CV1 quoted in what follows include a correction for the red leak of the UV filters. This was estimated using SYNPHOT, which is part of the STSDAS package in IRAF, combined with the newer filter-throughput data in ISR0907. ${ }^{4}$ For the purposes of comparison with catalogued values, we calculated the Johnson $(U-V)_{0}$ colours for the CV using the transformation in equation (8) and the zero-points in table 7 of Holtzman et al. (1995).

\subsubsection{X-ray observations}

Our X-ray data set consists of $16 \mathrm{ks}$ of archival Chandra/ACIS-S data, obtained from the Chandra Data Archive (see Table 1). The central region of the cluster where CV1 resides appears on the backilluminated S3 chip of the ACIS detector, close to the aimpoint of the telescope. Beginning with the level-1 pipeline-processed event lists, the data were reduced with the CIAO4.1 software to apply the latest calibration. The data were filtered on the energy range for which the ACIS detector is calibrated, $0.3-10 \mathrm{keV}$. Bad pixels and times of high background were removed using the pipelineproduced bad pixel file and good-time intervals. Source detection was carried out with the WAVDETECT algorithm, which correlates the data with a 'Mexican hat' wavelet function. A source appearing at $\alpha=18^{\mathrm{h}} 36^{\mathrm{m}} 24.71, \delta=-23^{\circ} 54^{\prime} 35^{\prime \prime} 6$ is consistent with M22 CV1: this position corresponds to a radial offset of $\sim 0.7$ arcsec from the HST position (see Section 2.1.2), which is within the uncertainties of the HST $(\sim 0.5 \mathrm{arcsec})$ and Chandra $(0.6 \mathrm{arcsec})$ coordinates. We extracted the source spectrum with the CIAO4.1-contributed script for point sources, PSEXTRACT. The detector response and effective-area files were extracted with the MKACISRMF and MKARF tools and binned on the same energy grid. A background spectrum was extracted from source-free regions neighbouring the object. The same instrument response files were used for the background as for the source. The source spectrum was grouped to include at least 20 net counts per

2 IRAF is distributed by the National Optical Astronomy Observatories, which are operated by the Association of Universities for Research in Astronomy, Inc., under cooperative agreement with the National Science Foundation.

${ }^{3} \mathrm{http} / / /$ purcell.as.arizona.edu/wfpc2_calib/

${ }^{4} \mathrm{http}: / /$ www.stsci.edu/hst/wfpc2/documents/isr/wfpc2_isr0907.html

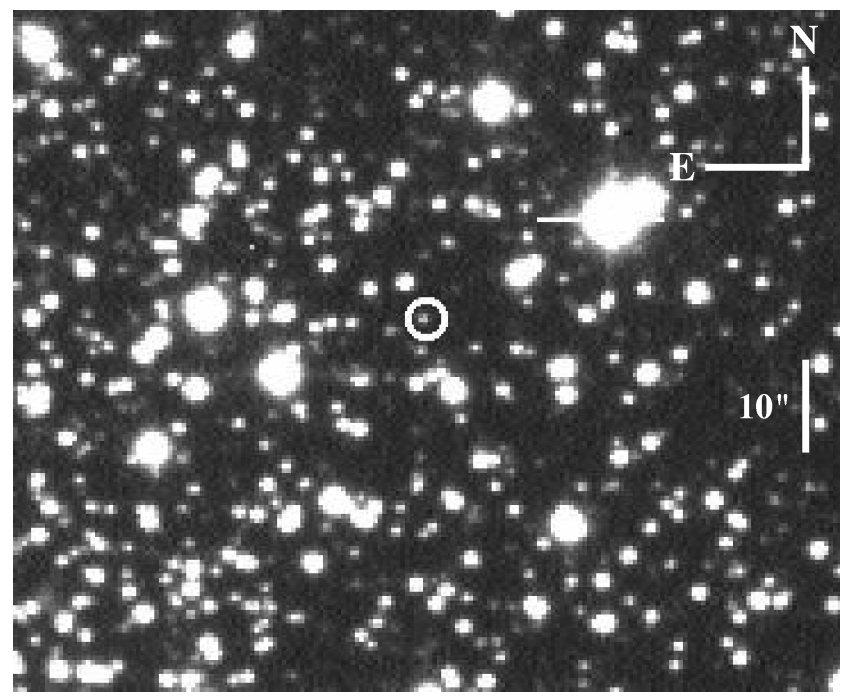

Figure 4. Finding chart for V101 in M5, taken from an archival INT $R$-band image.

bin. Fitting of the source and background spectra was performed in XSPEC version 12.4.

\subsection{M5: optical photometry and spectroscopy}

The optical spectroscopic observations of M5 were taken during 1991 July and August with the 4.2-m William Herschel Telescope (WHT) on La Palma, using both the red and blue arms of the Intermediate dispersion Spectrograph and Imaging System (ISIS). The R316R grating was used with the red arm and the R300B grating with the blue arm, producing a resolution of $3.3 \AA$. The seeing during the spectroscopic observations varied from 0.7 to 1.3 arcsec. The finding chart for the CV appears in Fig. 4.

We used two sets of imaging data. The first consists of $R$-band images obtained from the ING archive. ${ }^{6}$ The observations were taken with the 2.5-m Isaac Newton Telescope (INT) on La Palma over several nights in 1990 April and June. The uncertainty in the orbital period leads to a possible phase error of 10 per cent after 4 d. On combining data with a smaller temporal separation than this, the resulting data sets have approximately 80 per cent orbital phase coverage.

The second set of imaging data we obtained at the 2.4-m Hiltner telescope at Michigan-Dartmouth-MIT (MDM) observatory using a Sloan Digital Sky Survey $r$-band filter. It consists of 27 60-s exposures and 78 180-s exposures taken over four nights during 2009 July. The field of view was $4.97 \times 3.32 \operatorname{arcmin}^{2}$ while the images had a typical seeing of about $1.6 \mathrm{arcsec}$. This data set had approximately 50 per cent orbital phase coverage.

All images were prepared by debiasing, trimming and flatfielding with the CCDPROC routine in IRAF. For the spectral images, an aperture was selected around the target on each image and the spectrum was extracted using routines in the KPNOSLIT package in IRAF. The wavelength-calibration arc-lamp spectra $(\mathrm{CuNe}$ for the red and $\mathrm{CuAr}$ for the blue) were extracted using the same parameters as for the corresponding target spectra. The arc spectra were

\footnotetext{
${ }^{5} \mathrm{http}: / /$ heasarc.gsfc.nasa.gov/docs/xanadu/xspec/

${ }^{6}$ This paper makes use of data obtained from the Isaac Newton Group Archive which is maintained as part of the CASU Astronomical Data Centre at the Institute of Astronomy, Cambridge.
} 
fitted with a high-order cubic spline to remove the shape of the continuum of the illuminating arc-lamp. The wavelength solutions for the target spectra were calculated using the lines identified in the arc spectra and checked using the sky lines in a sky spectrum extracted from a source-free region of the spectral image containing the target. With only three blue spectra for which we had enough signal-to-noise ratio $(\mathrm{S} / \mathrm{N})$ to obtain a trace for extraction on the spectral image, we summed the spectra and removed cosmic rays (which were located on the continuum) manually in IRAF. We also summed the red spectra and again removed cosmic rays manually, checking for consistency against the median-combined cosmic ray filtered spectrum. Finally, we measured equivalent widths of any emission lines in the summed spectrum by fitting with a Gaussian line profile in IRAF, excluding from the fits data from any spectrum which had been affected by a cosmic ray hit on that emission line.

For the imaging data, profile-fitting photometry was performed with the DAOPHOT (Stetson 1987) package in IRAF. A set of bright, isolated stars is required to model the stellar profiles arising from the PSF of the light in an image. In a crowded field where no wellisolated stars are available, an iterative procedure is required to build the PSF. The following is the procedure we used to construct the PSF of our images. First, a Gaussian function was fitted to a sample of reasonably uncrowded stars with good $\mathrm{S} / \mathrm{N}$ and no defects, in an attempt to model the PSF. A constant PSF model was initially computed and fitted to the chosen PSF stars and their neighbours in order to subtract them from the image, revealing in the process previously invisible faint neighbouring stars - these were subsequently added to the star list, had photometry performed on them and were finally subtracted from the image. The process was iterated upon until a fairly complete list of PSF-star neighbours had been found and these were then subtracted from the original image to produce an image containing the PSF stars but excluding their close neighbours. From this image a PSF model which varied linearly across the image was computed. This new model was used to fit and subtract (more cleanly) the PSF stars and their neighbours from the original image in the same way as before, iterating on the process to eliminate the close neighbours of the PSF stars and finally produce an image containing only the PSF stars, which at this stage had become well-isolated enough to compute a sufficiently accurate model of the PSF to use for photometry. It was from this image that the final PSF model was made. This model was then fitted to the source (the CV, M5 V101) and several comparison stars to obtain their instrumental magnitudes. The process was repeated for each image and a light curve was constructed using the same brighter comparison star in each image (with constancy checked against several other comparison stars). The light curves were folded on the orbital period and plotted against orbital phase. Phase zero is arbitrary since the ephemeris was not known.

\section{M22 CV1: OUTBURSTS AND OPTICAL/X-RAY COLOURS}

\subsection{Optical variability}

Our IsIs light curve (Fig. 5) shows a $\sim 15$-d outburst in the $V$ band during 2004 May, the only large outburst seen in nine months of monitoring. The rise to maximum takes place over at least $5 \mathrm{~d}$, fading over another $10 \mathrm{~d}$, approximately. The actual peak of the outburst may have been missed in the $\sim 24$-h sampling gaps. We were unable to calibrate the outburst amplitude on the magnitude scale due to severe crowding effects combined with the apparent faintness of the $\mathrm{CV}$. The outburst was also caught in the $B$ band; however, due to the aforementioned difficulty in calibrating the flux, it is unclear if the lower $B$-band flux-levels compared to the $V$ band are real or not. The best available reference image to use for the $B$-band reductions came from the end of May when the flux level had more or less reached quiescent levels again - however, there may still have been some activity of the object at this time, which would, in effect,

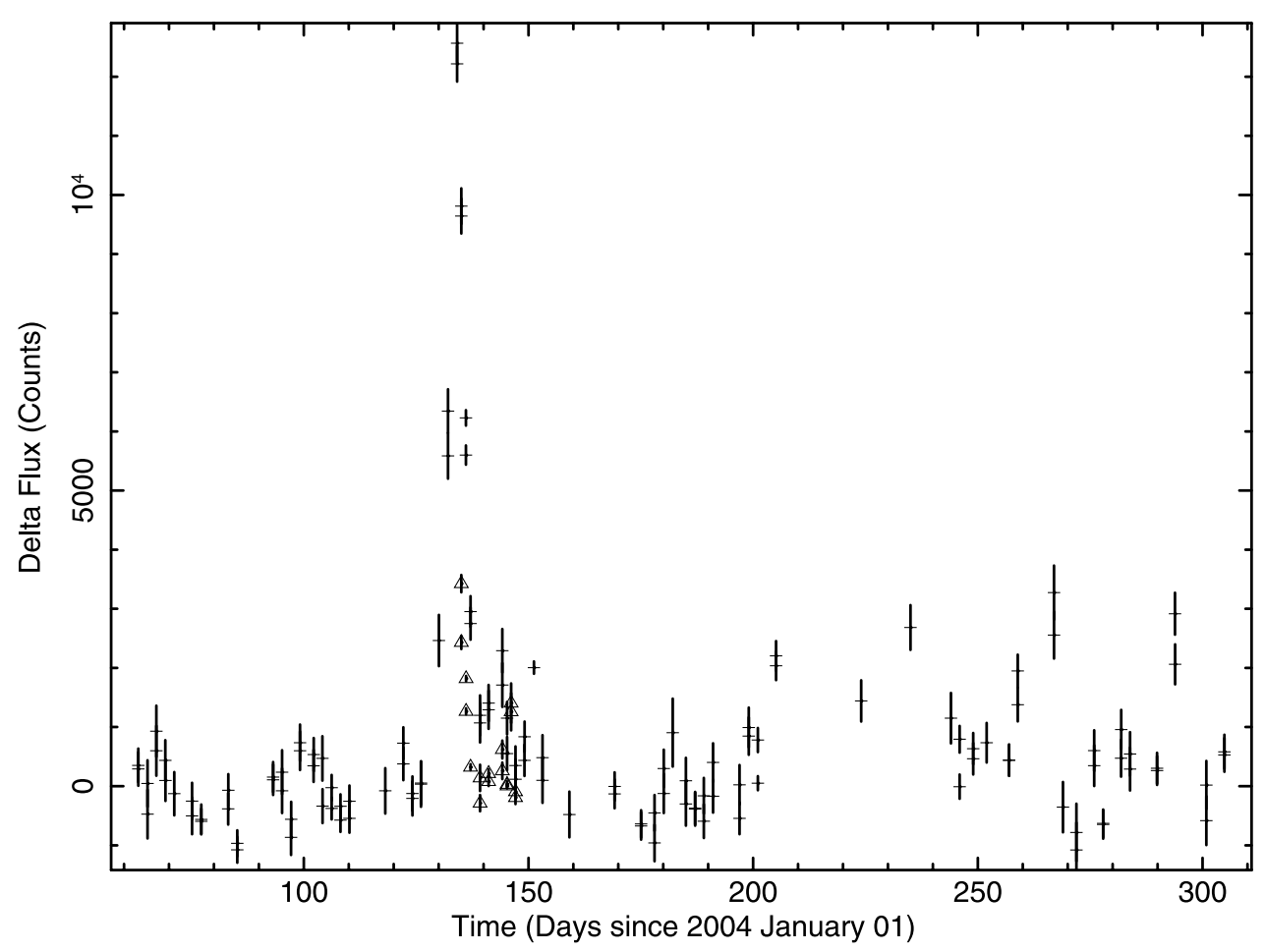

Figure 5. The IsIs differential flux light curve showing the 2004 May outburst. The crosses represent the $V$-band data and the triangles the $B$-band data. 
reduce the flux measured in the subtracted images. Inspecting the full data set, we see low-level flickering-like variability, typical of CVs, which appears to be present at a level above the noise - but again, caution is required in interpreting this feature without proper calibration.

Following the 1999 event recorded by Sahu et al. (2001), the system was observed in a bright state about once per year up until 2004. Pietrukowicz et al. (2005) estimated the outburst recurrence time for the system to be greater than $150 \mathrm{~d}$ based on their own observations as well as the 1999 event. Our observations are in agreement with this estimate. In nine months of monitoring we saw only one outburst of duration $\sim 15 \mathrm{~d}$. The sampling was every second night for most of the observing programme, but after discarding the images with the worst seeing, we are left with gaps in the data of up to $17 \mathrm{~d}$. This means there is a possibility we may have missed another similar outburst; also, there is a stronger likelihood of any shorter outbursts of only a few days duration - if present - having gone undetected.

\subsection{Optical colours}

In archival HST/WFPC2 data from several epochs covering the period 1994-2000, Anderson et al. (2003) found that the object initially identified by Sahu et al. (2001) as a microlensing event was 0.5-mag brighter through a $\mathrm{H} \alpha$ filter than main-sequence stars of the same magnitude. It was also a cluster member and coincident with a ROSAT source. This combination of factors led them to identify the object as the first known CV in the cluster (CV1). Interestingly, however, the broad-band $\left(B_{439}-R_{675}\right)$ and $\left(V_{606}-\right.$ $I_{814}$ ) colours place the star to the red side of the main sequence, in the region generally associated with detached main-sequence binaries. By contrast, CVs typically appear bluer than main-sequence stars owing to the presence of a hot accretion disc.

As discussed in Section 2.1.2, in order to further investigate the broad-band colours of CV1, we analysed archival HST images from 2006 taken in $n U V, U$ and $V$ filters. In the STMAG photometric system, we find $V_{555}=19.25 \pm 0.05$ for the $\mathrm{CV}$, with colours $\left(U_{336}-V_{555}\right)_{0}=0.19 \pm 0.12 \mathrm{mag}$ and $\left(n U V_{255}-U_{336}\right)_{0}=0.20 \pm$ $0.25 \mathrm{mag}$, assuming a foreground reddening of $E(B-V)=0.34$ (with an error of 10 per cent) in the direction of M22 (Harris 1996). (We have chosen the STMAG system because it is better calibrated than the synthetic WFPC2 system at UV wavelengths.) Converting to the Johnson system (see Section 2.1.2), our measurements show that at $(U-V)_{0}=0.13 \pm 0.13$ mag CV1 does not appear as UVbright as field CVs in quiescence, which typically have $(U-V)$ colours in the range from -0.1 to -0.7 mag (e.g. Bruch \& Engel 1994). This result is not wholly unexpected if we consider that the red colours found by Anderson et al. (2003) suggest that the secondary contributes significantly to the red optical light - perhaps down even as far as the $V$ band. If this is indeed the case, the optical emission of the donor would rival the UV emission from the disc, primary or hotspot, resulting in a less pronounced UV colour excess for the system than seen in typical field CVs. In any case, we still see that in the $U_{336}$ versus $\left(U_{336}-V_{555}\right)$ CMD CV1 does have a UV excess (of $\sim 0.4 \mathrm{mag}$ ) compared to the GC main sequence - as would be expected for CVs (see Fig. 6).

Using the $(U-V)_{0}$ colour to constrain the temperature, at a distance to M 22 of $3.2 \pm 0.3 \mathrm{kpc}$ (Harris 1996), we calculate $L_{\mathrm{UV}} \sim$ $2.2 \times 10^{32} \mathrm{erg} \mathrm{s}^{-1}$, over the UV wavelength range 2400-3400 $\AA$, for a blackbody spectrum of $\sim 9000 \mathrm{~K}$. The uncertainty in this value is of the order of 40 per cent.

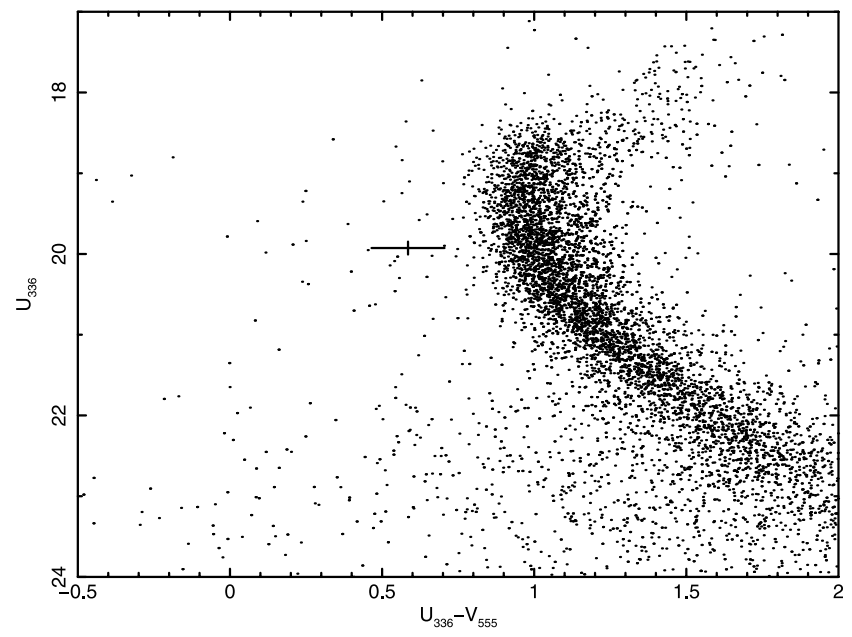

Figure 6. $U_{336}$ versus $\left(U_{336}-V_{555}\right)$ colour-magnitude diagram (CMD) for M22, with $U_{336}$ and $V_{555}$ observations both from the same epoch in $2006-$ see Table 1. The magnitudes are in the STMAG system. The location of the $\mathrm{CV}$ is marked with error bars.

\subsection{X-ray characteristics}

The X-ray spectrum (Fig. 7) is well fitted by a power law as well as other simple models such as a thermal bremsstrahlung. The parameters of the various fits are given in Table 2: the photon index we find for the power-law fit $(\Gamma=1.51 \pm 0.11)$ and the temperature of the Bremsstrahlung fit $\left(k T_{\text {Brems }}=10.00 \pm 4.07 \mathrm{keV}\right)$ are consistent with those found by Webb et al. (2004) $\left(\Gamma=1.70 \pm 0.13 ; k T_{\text {Brems }}=\right.$ $9.69 \pm 3.11 \mathrm{keV})$. Webb et al. also found an absorption line at $1 \mathrm{keV}$ in their XMM-Newton spectrum of the object. We checked for the presence of this line by adding a Gaussian absorption line at $1 \mathrm{keV}$. In contrast to the results of Webb et al., this did not improve the fit. Considering the relatively poor $\mathrm{S} / \mathrm{N}$ of our data, we also added a simulated absorption line at the same estimated normalization as that of Webb et al., to examine the effect on the fit. The addition significantly worsened the power-law fit, suggesting that no such line is present in our data.

The X-ray spectral-hardness ratio was calculated for CV1 from the Chandra data in the same manner as Heinke et al. (2005) using

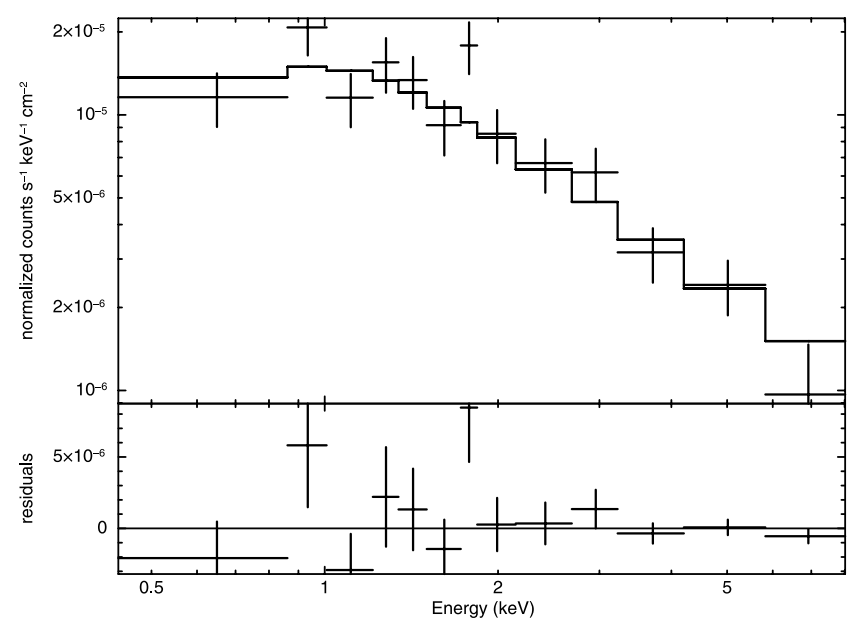

Figure 7. The Chandra/ACIS-S spectrum of M $22 \mathrm{CV} 1$ fitted with a powerlaw model (xSPEC model parameters: wabs(po), $N_{\mathrm{H}} \sim 2.2 \times 10^{21} \mathrm{~cm}^{-2}, \Gamma \sim$ $\left.1.5, \chi_{\text {reduced }}^{2}=1.12\right)$. The residuals of the fit are shown in the lower panel. 
Table 2. Parameters of the best-fitting spectral models to the Chandra/ACIS-S data for CV1. The flux given is the unabsorbed flux in the $0.3-10 \mathrm{keV}$ energy range. The hydrogen column was frozen to the value for the cluster $\left(2.2 \times 10^{21} \mathrm{~cm}^{-2}\right.$; Webb et al. 2004)

\begin{tabular}{ccccccc}
\hline $\begin{array}{c}N_{\mathrm{H}} \\
\left(\times 10^{21} \mathrm{~cm}^{-2}\right)\end{array}$ & Model & $k T(\mathrm{keV})$ & $\begin{array}{c}\text { Photon } \\
\text { index }\end{array}$ & $\chi^{2}$ & d.o.f. & $\begin{array}{c}\text { Flux } \\
\left(\times 10^{-14} \mathrm{erg} \mathrm{cm}^{-2} \mathrm{~s}^{-1}\right)\end{array}$ \\
\hline 2.2 & PL & - & $1.51 \pm 0.11$ & 1.12 & 11 & 21.5 \\
2.2 & Brems. & $10.00 \pm 4.07$ & - & 0.98 & 11 & 19.7 \\
2.2 & $\mathrm{RS}^{a}$ & $11.54 \pm 4.80$ & - & 1.22 & 11 & 21.2 \\
2.2 & PL+Gau. $^{b}$ & - & $1.61 \pm 0.11$ & 1.81 & 10 & \\
\hline
\end{tabular}

${ }^{a}$ Raymond Smith;

${ }^{b}$ line centre $=1.00 \pm 0.05, \sigma=0.1$, normalized to drop to 0.6 of continuum.

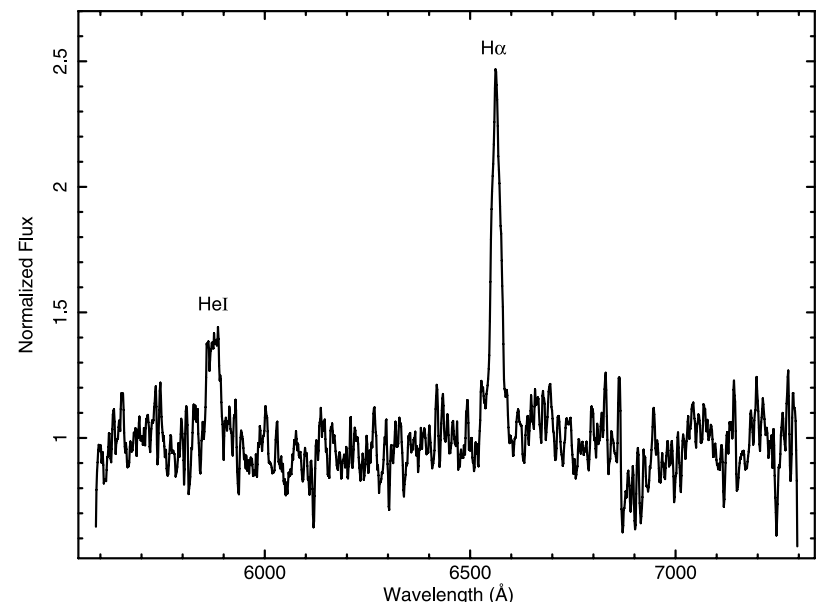

Figure 8. The red summed WHT/ISIS spectrum of M5 V101 in quiescence.

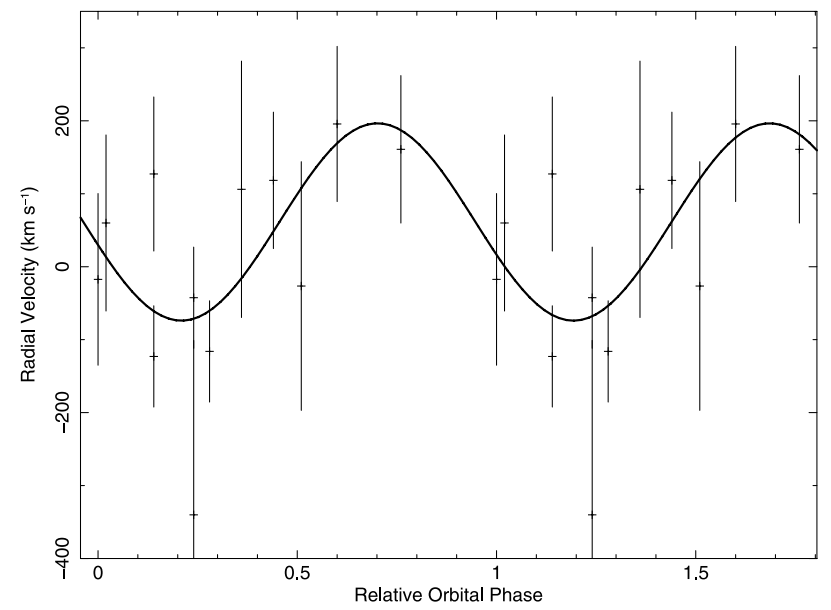

Figure 9. The radial velocities from the $\mathrm{H} \alpha$ emission-line fits folded on the orbital period of M5 V101.

the same equation they used for the plot in their Fig. 9:

$X_{\text {colour }}=2.5 \log \frac{\mathrm{F}_{\mathrm{X}[0.5-1.5 \mathrm{keV}]}}{\mathrm{F}_{\mathrm{X}[1.5-6 \mathrm{keV}]}}$.

The hardness of its X-ray colour, $X_{\text {colour }}=-0.39$, combined with the $\mathrm{X}$-ray luminosity we calculate in the $0.5-6 \mathrm{keV}$ band, $L_{\mathrm{X}[0.5-6 \mathrm{keV}]}=$ $1.4 \times 10^{32} \mathrm{erg} \mathrm{s}^{-1}$, places CV1 in the region of the plot of Heinke et al. occupied by the 47 Tuc CVs. By contrast, quiescent LMXBs have significantly softer X-ray colours (see e.g. fig. 10 of Heinke et al. 2005) even though their X-ray luminosities, though usually

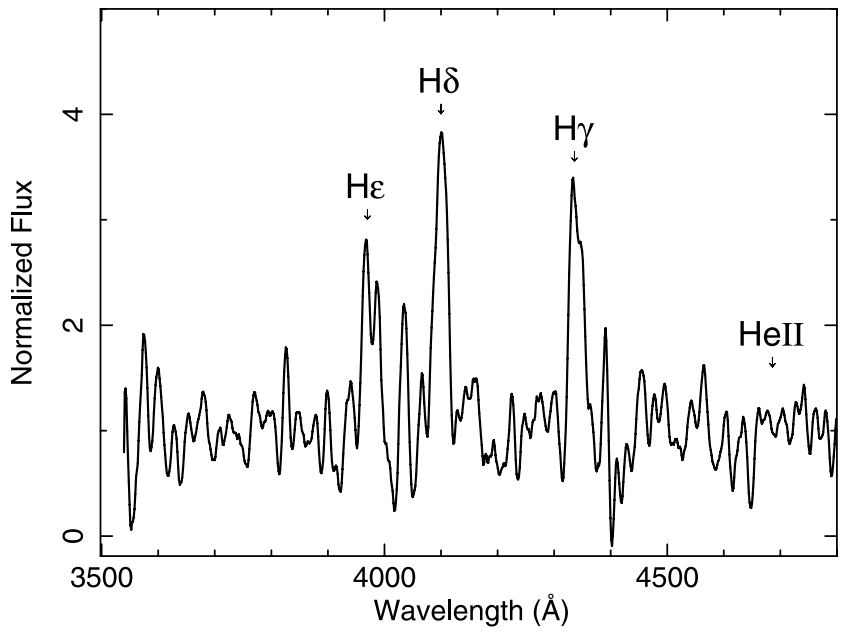

Figure 10. The blue summed WHT/ISIS spectrum of M5 V101 in quiescence.

higher, range down to a similar $\sim 10^{32} \mathrm{erg} \mathrm{s}^{-1}$. We conclude that the identification of this source as a $\mathrm{CV}$ is correct.

\section{M5 V101}

\subsection{Spectrum of M5 V101}

$\mathrm{H} \alpha \lambda 6563 \AA$ appears as the strongest emission line in the red spectra, with an equivalent width of $50 \pm 5 \AA$ in the summed spectrum (Fig. 8). He I $\lambda 5876 \AA$ also appears in emission with an equivalent width of $8.5 \pm 2 \AA$. The absorption appearing at $6867-6884 \AA$ is the Fraunhofer $B$ band caused by molecular $\mathrm{O}_{2}$ in the atmosphere. The weak absorption feature at $7118 \AA$ may be associated with the secondary star, but the origin of other apparent features, such as at 6118 and $7245 \AA$, cannot be identified (they do not seem to be telluric in nature, for example). The $\mathrm{H} \alpha$ emission line was fitted with a Gaussian profile in the individual spectra and the resulting radial velocity curve - phased on the 5.796-h orbital period (Neill et al. 2002) - is shown in Fig. 9. Despite the poor S/N, we find that the $\gamma$ velocity from the fit, $57 \pm 35 \mathrm{~km} \mathrm{~s}^{-1}$, is consistent with the cluster velocity of $52.6 \pm 0.4 \mathrm{~km} \mathrm{~s}^{-1}$ for M5 (Harris 1996).

The summed blue spectrum (Fig. 10) displays prominent Balmer emission but there is no evidence for any He II emission.

A list of all the emission line measurements is given in Table 3.

\subsection{Photometry}

Neill et al. (2002) found $P_{\text {orb }}=5.796 \pm 0.036 \mathrm{~h}$ for M5 V101 from $I$-band photometry. They saw two peaks in the orbital modulation 
Table 3. Characteristics of emission lines present in M5V101 spectrum.

\begin{tabular}{lccc}
\hline Line & $\begin{array}{c}\text { Wavelength } \\
(\AA)\end{array}$ & $\begin{array}{c}\text { Equivalent width } \\
(\AA)\end{array}$ & $\begin{array}{c}\text { Velocity FWHM } \\
\left(\mathrm{km} \mathrm{s}^{-1}\right)\end{array}$ \\
\hline $\mathrm{H} \alpha$ & 6563 & $50 \pm 3$ & $1100 \pm 150$ \\
$\mathrm{He} \mathrm{I}$ & 5876 & $22 \pm 5$ & $1800 \pm 100$ \\
$\mathrm{H} \gamma$ & 4340 & $90 \pm 30$ & $1900 \pm 200$ \\
$\mathrm{H} \delta$ & 4102 & $80 \pm 20$ & $1700 \pm 150$ \\
$\mathrm{H} \epsilon$ & 3970 & $60 \pm 30$ & $2300 \pm 300$ \\
\hline
\end{tabular}

in the redder $I$-band light curve and only one peak per orbit in the $V$ band, leading them to interpret one of the modulations as being associated with the secondary, which, at K5-M0 [which they estimated from their $(V-I)$ colour, $\simeq 2.2]$, would be much fainter in the $V$ band.

Our INT $R$-band light curve for the night of 1990 April 1 (Fig. 11a) exhibits a $0.3-\mathrm{mag}$ modulation at the orbital period of Neill et al. (2002). The modulation does not appear as strongly in the June data (Fig. 11b). Some flickering of the object was observed at this time. We superimposed the same model (equation 2) used by Neill et al.,

$y=a+b \sin (c \phi+d)+e \sin (f \phi+g) ;$

on our folded light curves for comparison: the resulting plots are shown in Fig. 11. The main peak we observe in the orbital light curve is probably due to the hotspot (the region where the accretion stream from the secondary impacts the accretion disc) rotating into view while the absence of any eclipse suggests the inclination is not high (Neill et al. estimated the inclination to be between $50^{\circ}$ and $60^{\circ}$ ). We find reasonable agreement with the results of Neill et al.: our $R$-band data show an intermediate situation between what they observed in the $V$ and $I$ bands, with a second but fainter peak visible in the orbital modulation.

The CV was on average $0.2 \pm 0.1$ mag brighter during the MDM observations than during the INT observations. Thus, it might be expected that the increased luminosity of the accretion disc would dilute the contribution from the secondary, removing the associated second peak - if such is its source - from the orbital modulation. Unfortunately, the phase coverage in our MDM $r$-band folded light curve (Fig. 11c) is insufficient to enable us to discern the morphology of the orbital modulation.

\subsection{X-ray characteristics}

The X-ray luminosity of the source in the $0.5-2.5 \mathrm{keV}$ band was calculated by Hakala et al. (1997) to be $L_{\mathrm{X}} \simeq 1.1 \times 10^{32} \mathrm{erg} \mathrm{s}^{-1}$. We extrapolate the flux they measured to find the flux in the $0.5-6 \mathrm{keV}$ band by fitting with a $3-\mathrm{keV}$ bremsstrahlung model in WebPIMMS, ${ }^{7}$ finding $F_{\mathrm{X}} \simeq 2.4 \times 10^{-14} \mathrm{erg} \mathrm{s}^{-1} \mathrm{~cm}^{-2}$ (unabsorbed). We calculate the UV and optical flux using the quiescent $V$-band magnitude of $20.27 \pm 0.04$ mag from Kaluzny et al. (1999) with the formula, $\log F_{\text {uv }+ \text { opt }}=-0.4 m_{\mathrm{V}}-4.32$, from van Teeseling, Beuermann \& Verbunt (1996), to find $F_{\text {uv }+ \text { opt }} \simeq 3.7 \times 10^{-13} \mathrm{erg} \mathrm{s}^{-1} \mathrm{~cm}^{-2}$. This leads to an X-ray-to-UV/optical flux ratio of $F_{\mathrm{X}} / F_{\mathrm{uv}+\mathrm{opt}} \sim 0.06$. Previously, the system was seen as faint as $V=22.5$ (Kukarkin \& Mironov 1970), which would lead to an $F_{\mathrm{X}} / F_{\text {uv }+ \text { opt }}$ ratio a factor of 10 higher, but there is no evidence for the system being this faint during the epoch from which we take the measurements that we use in our calculation. Nevertheless, since we cannot be sure that the

\footnotetext{
${ }^{7} \mathrm{http}: / /$ heasarc.nasa.gov/Tools/w3pimms.html
}
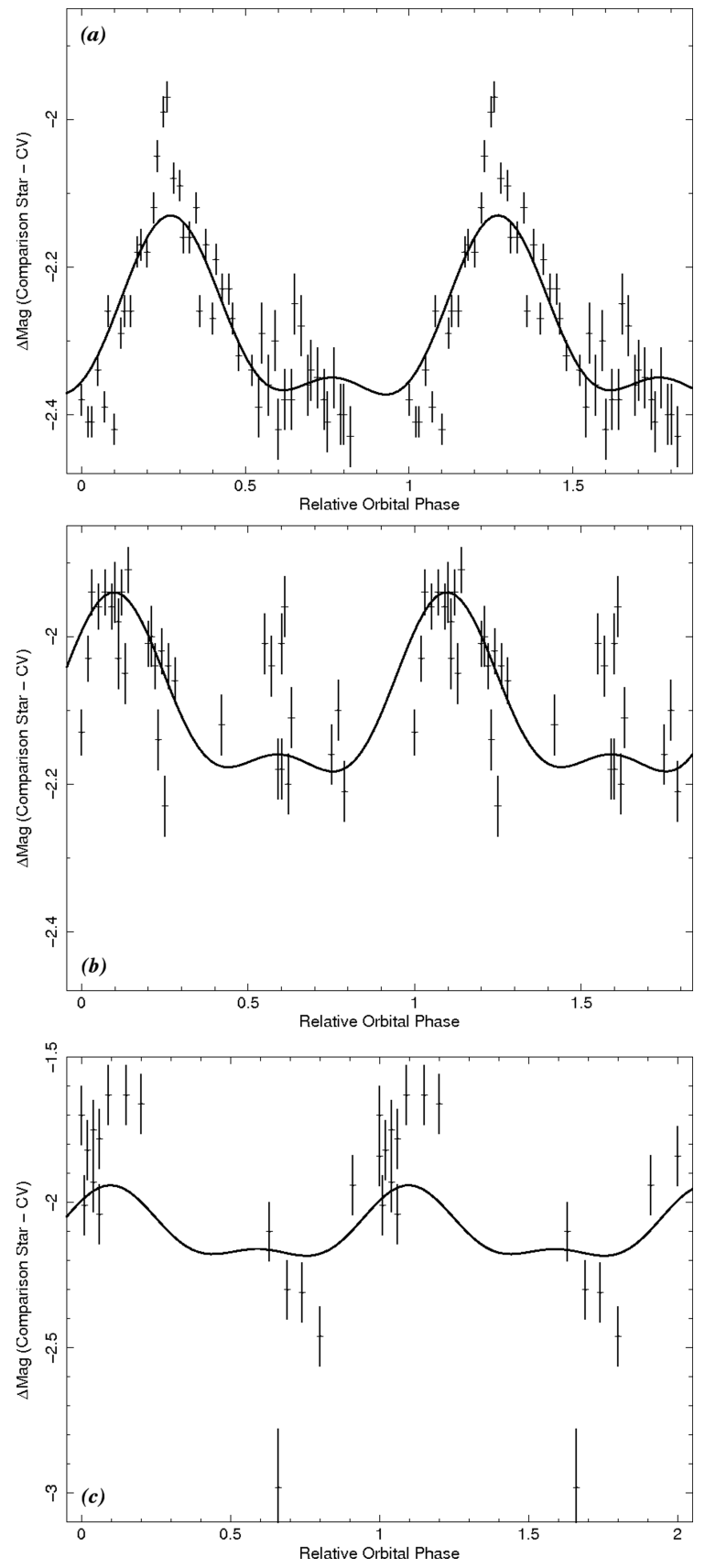

Figure 11. The M5 V101 light curves folded on the orbital period, $P_{\text {orb }}=$ $5.796 \pm 0.036$ h: (a) 1990 April INT $R$-band data; (b) 1990 June INT $R$-band data; (c) 2009 July MDM $r$-band data. We have superimposed the model of Neill et al. (2002) (see Section 4.2) which they fit to their $V$-band data, using their model parameters, on our folded light curves.

system was not fainter at the time the X-ray flux was measured, we consider the $F_{\mathrm{X}} / F_{\text {uv }+ \text { opt }}$ ratio estimated above to be a lower limit.

\section{DISCUSSION}

\subsection{M22 CV1}

\subsubsection{X-ray properties: CV or LMXB? Magnetic or non-magnetic?}

Previously Webb et al. (2004) found $\Gamma=1.70 \pm 0.13$ for a powerlaw fit to archival XMM-Newton X-ray spectral data for the source; 
in addition they found an even softer power-law photon index if they added a Gaussian absorption line at $1 \mathrm{keV}$, which improved their fit. They interpreted this absorption line as possible electron cyclotron resonance in a neutron star pulsar. The lack of any evidence for such an absorption line in our data weakens the case for a neutron star pulsar (or LMXB) identification for CV1. The hardness of the $\mathrm{X}$-ray colour of the object, $X_{\text {colour }}=-0.39$, is also more consistent with a CV than a quiescent LMXB. Thus, based on these X-ray properties of the system, it appears much more like a $\mathrm{CV}$ than a qLMXB.

We also calculated the X-ray luminosity in the Chandra/ACIS $0.3-10 \mathrm{keV}$ band, $L_{\mathrm{X}} \simeq 2.2 \times 10^{32} \mathrm{erg} \mathrm{s}^{-1}$, and found it is at the high end of the range of X-ray luminosities for non-magnetic DNe, but is typical for the magnetic intermediate polars (IPs). Our bestfitting power-law spectrum is relatively hard, with photon index $\Gamma=$ $1.51 \pm 0.11$. Heinke et al. (2008) investigated the relationship between CV subtype and X-ray spectrum and luminosity for a sample of CVs comprising confirmed magnetic CVs (IPs and polars), quiescent DN and nova-like CVs (NL) and GC CVs. They found the distribution of photon index differed significantly between magnetic CVs, which are harder X-ray sources (mean $\Gamma=1.22, \sigma=0.33$ ), and non-magnetic CVs (mean $\Gamma=1.97, \sigma=0.20$ ), but was fairly consistent within either group. The cluster sources were found to comprise $\sim 40$ per cent magnetic systems, based on the photon index distribution. Our measured photon index of $\Gamma=1.51 \pm 0.11$ places $\mathrm{CV} 1$ on the borderline between the magnetic and non-magnetic distributions. However, the combination of our result with the photon index result of Webb et al. (2004), which, although consistent with our value, ranges to softer values, tips the balance slightly in favour of a non-magnetic CV classification for the object.

\subsubsection{Outburst characteristics}

In the optical, the characteristics of the $\sim 15-\mathrm{d} 2004$ May outburst of M22 CV1 covered by our observations are consistent with previous reports of 15-20 d, 2-3 mag eruptions of the system by Sahu et al. (2001), Bond et al. (2005) and Pietrukowicz et al. (2005). These outburst characteristics are also consistent with normal outbursts of DN, which typically have durations from 2-20 d (Warner 1995), where this duration is also correlated with the recurrence time for outbursts. Pietrukowicz et al. (2005) estimated the outburst recurrence time for $\mathrm{CV} 1$ to be greater than $150 \mathrm{~d}$ - our observations are consistent with this limit.

The typical duration of outbursts of CV1, at 15-20 d, would tend to favour a longer orbital period estimate of the order of $10 \mathrm{~h}$ or more (e.g. Ak, Ozkan \& Mattei 2002). In addition, the outburst properties of CV1 are not unlike those of non-magnetic DNe of longer orbital period, such as BV Cen, which undergoes outbursts with an amplitude of $\sim 3$ mag and a 30-d duration separated by $150 \mathrm{~d}$ (although these tend to be more symmetrical in rise and decay than those of CV1).

If magnetic, CV1 cannot be a polar because their absent discs preclude outbursts. On the other hand, IPs - with their partially truncated inner discs - do exhibit outbursts. However, the outburst characteristics of CV1 are not consistent with any particular example of the known IPs. GK Per, with its infrequent outbursts which persist for two months, remains an anomalous system. Other IP systems such as EX Hya exhibit a similar outburst amplitude to CV1, $\sim 3.5 \mathrm{mag}$, but these outbursts last only $2-3 \mathrm{~d}$ and have a longer recurrence time of $\sim 2 \mathrm{yr}$ (Hellier et al. 1989), while IPs such as TV Col and V1223 Sgr show even shorter duration low-amplitude outbursts that last only $\sim 0.5 \mathrm{~d}$. Thus, on the basis of its outburst properties, CV1 does not resemble a magnetic system. We can also eliminate the SU UMa class based on the morphology of the outburst light curve: even though our sampling is too sparse to detect superhumps (the modulation which is the defining characteristic of the SU UMa class) in the outburst light curve, we find no evidence for the extended, sloping plateau of brightness associated with superoutbursts. Similarly, Pietrukowicz et al. (2005) found no evidence for the presence of superhumps in the 2000 August outburst light curve. This leaves the longer period $U$ Gem-type $\mathrm{DN}$ as the group of CVs with the most similar properties to CV1 (e.g. BV Cen).

\subsubsection{Optical colours}

Echevarría \& Jones (1984) show that for a sample of field DN, the $(B-V)$ and $(U-B)$ colours are well correlated with orbital period, with those systems having $P_{\text {orb }}<5.5 \mathrm{~h}$ being significantly bluer than those with $P_{\text {orb }}>7 \mathrm{~h}$. Conversely, for the same sample, the $(V-R)$ and $(R-I)$ colours do not appear to be well correlated with $P_{\text {orb }}$. Based on its $(U-V)_{0}$ colour of $0.13 \pm 0.13 \mathrm{mag}, \mathrm{CV} 1$ appears to be consistent with having $P_{\text {orb }}>7 \mathrm{~h}$. The blackbody temperature of $\sim 9000 \mathrm{~K}$ inferred from the $(U-V)_{0}$ colour is consistent with emission from the accretion disc.

Anderson et al. (2003) found that the $\left(B_{439}-R_{675}\right)$ and $\left(V_{606}-\right.$ $I_{814}$ ) colours of $\mathrm{CV} 1$ are unusually red for a $\mathrm{CV}$, about 0.2 and 0.1 mag redwards of the main sequence, respectively. A possible explanation for these red colours, previously mentioned by Anderson et al., is that a secondary larger than a normal main-sequence star dominates the optical emission of the system. Baraffe \& Kolb (2000) show that for longer orbital period ( $P_{\text {orb }} \gtrsim 6 \mathrm{~h}$ ) CVs, the degree of nuclear evolution of the secondary is crucial in setting its spectral type, with the most evolved donors having spectral types significantly later than their main-sequence analogues. Some degree of nuclear evolution of the donor off the main sequence could explain the location of CV1 in the CMDs of Anderson et al. An enhanced contribution to the $V$ band from such a secondary might also explain the relatively low UV excess inferred from the $(U-$ $V$ ) colour (see Section 3.2). Although an established example of an evolved GC CV secondary may be found in the DN AKO 9 in 47 Tuc (see the CMDs of Albrow et al. 2001; Edmonds et al. 2003a and the calculations of Knigge et al. 2003), the ( $V-I)$ colours of many other GC CVs (e.g. Cool et al. 1998; Edmonds et al. 2003a) suggest a lesser, if any, degree of nuclear evolution of the donors in these systems.

We note that the location of CV1 on the $\left(B_{439}-R_{675}\right)$ and $\left(V_{606}-\right.$ $I_{814}$ ) CMDs of Anderson et al., together with its UV and X-ray colours, could also be explained if CV1 were part of a hierarchical triple system in which the third star were a main-sequence star of mass comparable to the secondary star in CV1. It would be of interest to determine whether such a system could survive in the core of M22. Alternatively, the $\left(B_{439}-R_{675}\right)$ and $\left(V_{606}-I_{814}\right)$ colours could be accounted for by the presence of a line-of-sight main-sequence star. The relatively low density of stars in the vicinity of CV1 in the HST image makes this unlikely, but it cannot be ruled out.

\subsubsection{X-ray to optical/UV flux ratio: the CV subclass of CVI}

A useful discriminant of the subtype of a $\mathrm{CV}$ is the ratio of X-ray to UV and/or optical flux. 
Verbunt et al. (1997) demonstrated that the different classes of $\mathrm{CV}$ can be subdivided as follows: SU UMa and U Gem-type CVs, $F_{\mathrm{X}} / F_{\mathrm{uv}+\mathrm{opt}} \sim 0.1 ; F_{\mathrm{X}} / F_{\mathrm{uv}+\mathrm{opt}} \sim 0.01$ for Z Cam-type CVs; finally, UX UMa-type (or novalike) $\mathrm{CVs}$ have the lowest ratios at $F_{\mathrm{X}} / F_{\text {uv }+ \text { opt }} \sim 0.001$.

Calculating the ratio of X-ray to UV plus optical flux in an equivalent manner for $\mathrm{CV} 1$, we find $F_{\mathrm{X}} / F_{\text {uv+opt }} \sim 0.07$ [where the discrepancy with the result of Anderson et al. (2003) arises from the inclusion of the UV flux here]. This value again suggests a $U$ Gem classification for CV1.

\subsubsection{X-ray to optical/UV flux ratio: constraining the orbital period of $C V 1$}

The orbital period distribution of CVs typically ranges from tens of minutes up to about $15 \mathrm{~h}$. The value of $P_{\text {orb }}$ for a CV can reveal much about the parameters of the system (e.g. Warner 1995).

Whilst it is challenging to directly measure the orbital period for a system as faint and crowded as CV1 in M22, we can none the less attempt to constrain it by comparing the optical, UV and X-ray properties of the system to those of Galactic CVs with known orbital periods. For example, the absolute magnitude at minimum of the source, $M_{\mathrm{V}} \sim 5.4 \mathrm{mag}$ (Anderson et al. 2003), suggests an orbital period of $P_{\text {orb }}=13 \pm 3 \mathrm{~h}$ [see equation (18) of Warner 1987].

Furthermore, because of the increased UV luminosity of CVs at longer orbital periods due to their correspondingly higher accretion rates (van Teeseling et al. 1996), and the lack of any clear correlation between X-ray luminosity and orbital period, it would seem reasonable to expect a correlation between $F_{\mathrm{X}} / F_{\mathrm{uv}+\mathrm{opt}}$ and orbital period for non-magnetic CVs. In fact, van Teeseling et al. have found evidence for such a correlation: their fig. 6 shows that for a sample of non-magnetic field CVs, with increasing orbital period, there is a trend of decreasing $F_{\mathrm{X}} / F_{\mathrm{uv}+\mathrm{opt}}$.

Interestingly, however our $F_{\mathrm{X}} / F_{\mathrm{uv}+\text { opt }}$ value for CV1 places it in the region of the van Teeseling et al. plot with $P_{\text {orb }} \lesssim 2 \mathrm{~h}$, in contradiction to our previous orbital-period constraints. We discuss below two ways in which this could be resolved.

(1) Taking the contradiction with the van Teesling results at face value, one way to accommodate such a short orbital period would be to invoke the presence of a cooler (redder) line-of-sight interloper or a companion in a triple system. Then the intrinsically fainter, bluer $\mathrm{CV}$ would be more consistent with a shorter $P_{\text {orb }}$. In order to revise the $P_{\text {orb }}$ estimate using the relationship from Warner (1987) down to $2 \pm 1 \mathrm{~h}$, an absolute magnitude at minimum as faint as $\sim 9 \mathrm{mag}$ would be required. However, given the measured $\mathrm{H} \alpha$ excess of 0.54 mag (Anderson et al. 2003), a fainter CV would have a larger $\mathrm{H} \alpha$ excess translating to an unfeasibly large equivalent width of $\sim 330 \AA$ for the emission line, making this an unlikely explanation.

(2) A more likely explanation comes from a closer examination of the data used by van Teeseling et al. (1996): none of these systems is, in fact, U Gem types [the solitary datum of this class, EI UMa, has since been reclassified as an IP (Ramsay et al. 2008)].

Hence, we conclude that our classification of $\mathrm{CV} 1$ as a $\mathrm{DN}$ of $\mathrm{U}$ Gem type remains secure.

\subsection{M5 V101}

Strong He II $\lambda 4686-\AA$ line emission is characteristic of magnetic CVs (in polars, it is comparable in strength to $\mathrm{H} \beta$; Warner 1995). In IPs, for example, this high-excitation line is most likely powered by X-ray heating of the regions of the accretion curtains close to the magnetic poles, interior to the inner edge of the magnetically truncated accretion disc (Saito et al. 2010). The strong He I, $\mathrm{H} \alpha$ and Balmer emission (see Figs. 8 and 10) of V101 are characteristic of CVs in quiescence, while the lack of any obvious He II emission an upper limit of $6 \AA$ can be placed on the equivalent width of any He II $\lambda 4686$ - $\AA$ emission in the spectrum - suggests that the system is unlikely to be magnetic.

The enhanced contribution of the accretion disc to the luminosity of the system at shorter wavelengths means we would expect the absorption lines of the secondary star to be more apparent at longer wavelengths. The only possible absorption feature from the secondary which can be identified in our summed spectrum is a weak feature at $7118 \AA$, although we cannot be certain this is not an artefact.

Our phased optical light curves in the $R$ and $r$ bands show varying degrees of modulation at the orbital period of Neill et al. (2002). The main peak in the light curve may be due to the hotspot (see e.g. Wood et al. 1986). Comparing our $F_{\mathrm{X}} / F_{\mathrm{uv}+\mathrm{opt}} \geq 0.06$ to the trend in fig. 6 of van Teeseling et al. (1996), as we did for M22 CV1, again we find that for our result, their trend would predict a shorter orbital period of up to a maximum of around $3 \mathrm{~h}$, compared to the measured $P_{\text {orb }}=5.796 \pm 0.036 \mathrm{~h}$ (Neill et al. 2002). This discrepancy is not as pronounced as what we found for the $\mathrm{M} 22 \mathrm{CV}$, although in that case we did not have a measured $P_{\text {orb }}$ to compare with. However, again we note the lack of U Gem-type systems in the van Teeseling et al. (1996) sample.

\section{SUMMARY}

For M22 CV1, the X-ray properties confirm a CV rather than an LMXB nature. The high $L_{X}$ and X-ray spectral hardness are indicative of either a quiescent DN or an IP. The outburst characteristics are consistent with a normal long $P_{\text {orb }}(\gtrsim 10$ h) DN of U Gem-type, but cannot be very easily reconciled with any particular example of the known IPs. We find the expected UV excess compared to the cluster main sequence in $U_{336}$ versus $\left(U_{336}-V_{555}\right)$, and that the $(U-V)$ colour is consistent with a longer orbital period.

For the M5 CV, V101, our optical spectra are typical for a quiescent DN. The lack of any significant He II emission suggests the system is not magnetic. The modulation we observe in the $R$-band light curve is consistent with the orbital period of $5.796 \pm 0.036 \mathrm{~h}$ found by Neill et al. (2002). As this remains the GC CV most amenable to detailed study from the ground in quiescence, it will benefit from future higher $\mathrm{S} / \mathrm{N}$ phase-resolved optical spectroscopy.

\section{ACKNOWLEDGMENTS}

We would like to thank the referee, Christian Knigge, for helpful comments and suggestions. This research has made use of data obtained from the Chandra Data Archive and software provided by the Chandra X-ray Center ( CXC) in the application package CIAO. This research has also made use of the SIMBAD data base, operated at CDS, Strasbourg, France.

\section{REFERENCES}

Ak T., Ozkan M. T., Mattei J. A., 2002, A\&A, 389, 478

Alard C., 2000, A\&AS, 144, 363

Alard C., Lupton R. H., 1998, ApJ, 503, 325

Albrow M. D., Gilliland R. L., Brown T. M., Edmonds P. D., Guhathakurta P., Sarajedini A., 2001, ApJ, 559, 1060 
Anderson J., Cool A. M., King I. R., 2003, ApJ, 597, 137

Baraffe I., Kolb U., 2000, MNRAS, 318, 354

Bond I. A. et al., 2005, ApJ, 620, 103

Bruch A., Engel A., 1994, A\&AS, 104, 79

Cohn H. N. et al., 2010, ApJ, 722, 20

Cool A. M., Grindlay J. E., Krockenberger M., Bailyn C. D., 1993, ApJ, 410, L103

Cool A. M., Grindlay J. E., Cohn H. N., Lugger P. M., Slavin S. D., 1995, ApJ, 439, 695

Cool A. M., Grindlay J. E., Cohn H. N., Lugger P. M., Bailyn C. D., 1998, ApJ, 508, L75

Echevarría J., Jones D. J. P., 1984, MNRAS, 206, 919

Edmonds P. D., Gilliland R. L., Heinke C. O., Grindlay J. E., 2003a, ApJ, 596,1177

Edmonds P. D., Gilliland R. L., Heinke C. O., Grindlay J. E., 2003b, ApJ, 596,1197

Gänsicke B. T., 2005, in Hameury J.-M., Lasota J.-P., eds, ASP Conf. Ser. Vol. 330, The Astrophysics of Cataclysmic Variables and Related Objects. Astron. Soc. Pac., San Francisco, p. 3

Grindlay J. E., Hertz P., Steiner J. E., Murray S. S., Lightman A. P., 1984, ApJ, 282, L13

Grindlay J. E., Cool A. M., Callanan P. J., Bailyn C. D., Cohn H. N., Lugger P. M., 1995, ApJ, 455, 47

Hakala P. J., Charles P. A., Johnston H. M., Verbunt F., 1997, MNRAS, 285, 693

Harris W. E., 1996, AJ, 112, 1487

Heinke C. O., Grindlay J. E., Edmonds P. D., Cohn H. N., Lugger P. M., Camilo F., Bogdanov S., Freire P. C., 2005, ApJ, 625, 796

Heinke C. O., Ruiter A. J., Muno M. P., Belczynski K., 2008, in Bandyopadhyay R. M., Watcher S., Gelino D., Gelino C. R., eds, AIP Conf. Ser. Vol. 1010, A Population Explosion: The Nature \& Evolution of X-ray Binaries. Astron. Soc. Pac., San Francisco, p. 136

Hellier C., Mason K. O., Smale A. P., Corbet R. H. D., O’Donoghue D., Barrett P. E., Warner B., 1989, MNRAS, 238, 1107

Holtzman J. A., Burrows C. J., Casertano S., Hester J. J., Trauger J. T., Watson A. M., Worthey G., 1995, PASP, 107, 1065
Hut P., Verbunt F., 1983, Nat, 301, 587

Ivanova N., Heinke C. O., Rasio F. A., Taam R. E., Belczynski K., Fregeau J., 2006, MNRAS, 372, 1043

Kaluzny J., Thompson I., Krzeminski W., Pych W., 1999, A\&A, 350, 469

Knigge C., Zurek D. R., Shara M. M., Long K. S., Gilliland R. L., 2003, ApJ, 599, 1320

Kukarkin B. V., Mironov A. V., 1970, Astron. Zhurnal, 47, 1211

Neill J. D., Shara M. M., Caulet A., Buckley D. A. H., 2002, AJ, 123, 3298

Ó Tuairisg S., Butler R. F., Shearer A., Redfern R. M., Butler D., Penny A., 2003, MNRAS, 345, 960

Pietrukowicz P., Kaluzny J., Thompson I. B., Joroszyński M., Schwarzenberg-Czerny A., Krzeminski W., Pych W., 2005, Acta Astron., 55, 261

Pietrukowicz P., Kaluzny J., Schwarzenberg-Czerny A., Thompson I. B., Pych W., Krzeminski W., Mazur B., 2008, MNRAS, 388, 1111

Ramsay G., Wheatley P. J., Norton A. J., Hakala P., Baskill D., 2008, MNRAS, 387, 1157

Sahu K. C., Casertano S., Livio M., Gilliland R. L., Panagia N., Albrow M. D., Potter M., 2001, Nat, 411, 1022

Saito R. K., Baptista R., Horne K., Martell P., 2010, AJ, 139, 2542

Shara M., Bergeron L., Gilliland R., Saha A., Petro L., 1996, ApJ, 471, 804

Stetson P. B., 1987, PASP, 99, 191

van Dokkum P. G., 2001, PASP, 113, 1420

van Teeseling A., Beuermann K., Verbunt F., 1996, A\&A, 315, 467

Verbunt F., Bunk W. H., Ritter H., Pfeffermann E., 1997, A\&A, 327, 602

Warner B., 1987, MNRAS, 227, 23

Warner B., 1995, Cataclysmic Variable Stars. Cambridge Univ. Press, Cambridge

Webb N. A., Serre D., Gendre B., Barret D., Lasota J. -P., Rizzi L., 2004 A\&A, 424, 133

Wood J., Horne K., Berriman G., Wade R., O’Donoghue D., Warner B., 1986, MNRAS, 219, 629

This paper has been typeset from a $\mathrm{T}_{\mathrm{E}} \mathrm{X} / \mathrm{LT}_{\mathrm{E}} \mathrm{X}$ file prepared by the author. 